\title{
Mitteilungen des GRC
}

\section{GRC}

GERMAN RESUSCITATION COUNCIL

DEUTSCHER RAT FÜR WIEDERBELEBUNG

Notfall Rettungsmed 2020 $23: 236-237$

https://doi.org/10.1007/s10049-020-00701-y

() Springer Medizin Verlag GmbH, ein Teil von Springer Nature 2020
Deutscher Rat für Wiederbelebung -

German Resuscitation Council (GRC) e.V.

c/o Sektion Notfallmedizin, Universitätsklinikum Ulm

Prittwitzstraße 43, 89070 Ulm

info@grc-org.de

www.grc-org.de

Redaktion

Prof. Dr. B. W. Böttiger (V.i.S.d.P.)

B. Gliwitzky (V.i.S.d.P.)

B. Brock

N. Rott

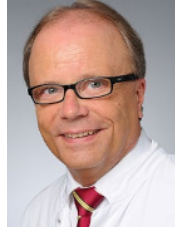

$\Delta$ Univ.-Prof.

Dr. med. Bernd

W. Böttiger,

Vorstandsvor-

sitzender GRC
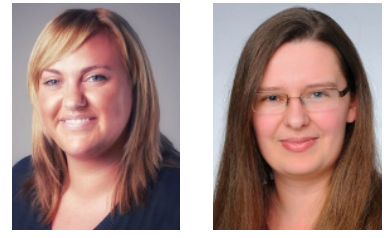

$\Delta$ Britta Brock Marketing GRC

$\Delta$ Nadine Rott, Geschäftsstelle GRC

\section{SAVE THE DATE - GRC Reanimationsdialog 2020}

Der erste GRC Reanimationsdialog fand 2019 in Leipzig statt und war ein voller Erfolg. Wir möchten gerne daran anknüpfen und Sie sehr herzlich zum zweiten GRC Reanimationsdialog vom 30.-31.10.2020 in Köln einladen. Es erwartet Sie auch wieder ein wissenschaftliches Programm mit vielen verschiedenen Vorträgen. Bitte notieren Sie sich bereits heute den Termin. In Kürze erhalten Sie mehr Informationen zum nächsten GRC Reanimationsdialog.

\section{Reanimationsleitlinien wegen COVID-19-Krise verschoben} jetzigen Zeit

\section{Stellungnahme des GRC zur Durchführung von Wiederbelebungsmaßnahmen im Umfeld der COVID-19-Pandemie}

Die COVID-19-Pandemie hat in den vergangenen Wochen erhebliche Veränderungen und Herausforderungen in der medizinischen Versorgung zur Folge gehabt. Hiervon besonders betroffen ist auch der Themenbereich der kardiopulmonalen Reanimation. Dies ist dadurch begründet, dass der akute Herz-Kreislaufstillstand unmittelbare BLS- bzw. ALS-Maßnahmen erfordert, gleichzeitig jedoch ein maximaler Schutz der hilfeleistenden Laien oder des medizinischen Fachpersonals in der gegebenen COVID-19-Situation sichergestellt werden muss.

Der Vorstand und das Exekutivkomitee des GRC haben diese Fragen vor dem Hintergrund des aktuellen internationalen Stand des Wissens ausführlich diskutiert und die daraus abzuleitenden Konsequenzen in einer Stellungnahme und Abbildung zusammengefasst. Sie können dies auf der GRC Homepage finden: www.grc-org.de/ueberuns/aktuelles/130-Stellungnahme-des-GRC-zur-Durchfuhrung-vonWiederbelebungsmassnahmen-im-Umfeld-der-COVID-19-Pandemie
Angesichts der aktuellen COVID-19-Pandemie hat der ERC beschlossen, die Veröffentlichung der Reanimationsleitlinien auf $2021 \mathrm{zu}$ verschieben.

Diese Entscheidung wurde getroffen, weil die meisten der am ERCLeitlinienprozess teilnehmenden Kollegen sehr stark in der COVID19-Krise involviert sind und weil im Moment ganz andere Themen im Vordergrund stehen.

Wir bedanken uns für das Verständnis.

\section{Werden Sie Mitglied beim GRC}

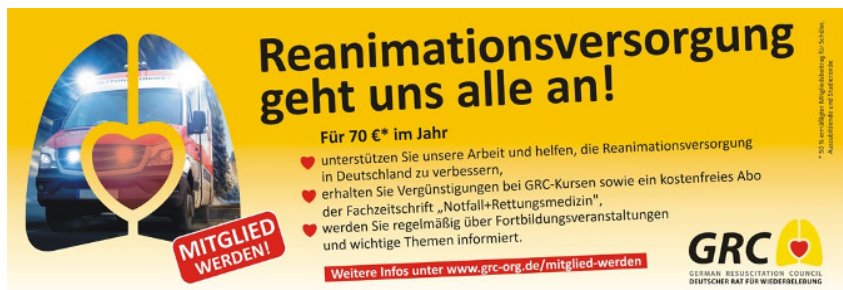




\section{GRC Kurszentrum in Fürth etabliert ALS Kurse in Uganda}

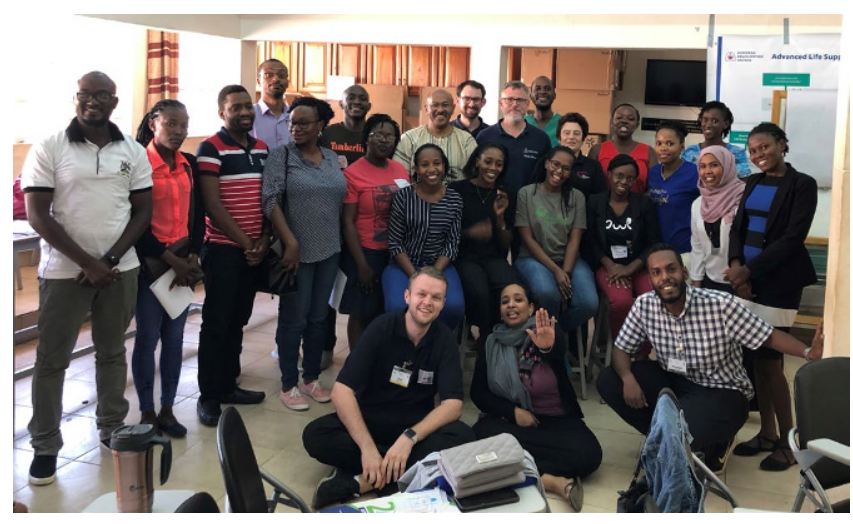

Die Kursformate des ERC/GRC sind ein wichtiges Instrument Fachkräfte im Gesundheitswesen in den Leitlinien der Versorgung von Patientinnen und Patienten mit Herzstillstand zu trainieren. Deutschland hat in den vergangenen Jahren von der flächendeckenden Verbreitung dieser Formate profitiert. In vielen Entwicklungs- und Schwellenländern existieren jedoch noch keine qualitativen Fortbildungskonzepte für Gesundheitspersonal zum Thema Wiederbelebung. Diese Erkenntnis führte dazu, dass die Arbeitsgemeinschaft Notfallmedizin Fürth zusammen mit dem ERC ein Projekt in Uganda initiierte. Die Arbeitsgemeinschaft Notfallmedizin Fürth e.V. (AGNF) organisiert als Kurszentrum Fürth seit 2007 das GRC Kurswesen im Raum Nordbayern.

Während eines ersten Besuches vor Ort erkannten Klaus Meyer, Sprecher des Kurskomitees des GRC und Vorsitzender der AGNF, und Ines Voigt, Projektleiterin der AGNF, den Mangel an leitlinienorientiertem und strukturiertem Training in Wiederbelebung vor Ort. Gefördert durch die Gesellschaft für Internationale Zusammenarbeit (GIZ) gelang es der AGNF ein Projekt zusammen mit dem ERC und der Makerere University in Kampala zu initiieren, das im Oktober 2019 gestartet werden konnte.

In den kommenden zwei Jahren sollen nun ca. 200 Anwender im Kursformat ALS geschult und Instruktoren ausgebildet werden. Nach Projektende sollen die Kolleginnen und Kollegen vor Ort in der Lage sein, die Kurse eigenverantwortlich zu organisieren. In einer ersten Projektphase im Januar 2020 wurden in einer ersten Kursreihe bereits 110 Anwender geschult und 15 Instruktorinnen und Instruktoren ausgebildet. In diesem Jahr sollen, in bis zu 9 weiteren Kursen, ca. 150-200 weitere Anwender geschult werden. Damit wäre das Projektziel bereits nach einem Jahr mehr als erreicht.

Unterstützt wurden die deutschen Kolleginnen und Kollegen von

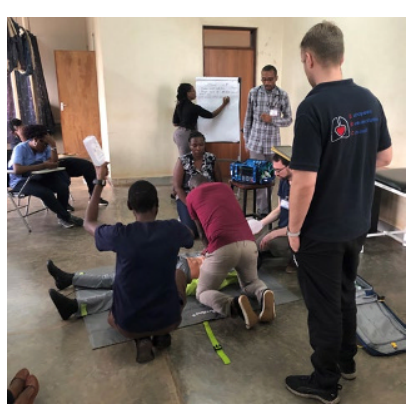
Instruktoren aus dem Sudan und aus Malta. Meyer berichtet, dass die Erfahrungen aus den Kursen auch für die deutschen Kolleginnen und Kollegen, die eine hochspezialisierte und hochtechnisierte Arbeitsumgebung gewohnt sind, sehr wertvoll waren. Nicht alles, was zu einer professionellen Versorgung von Patientinnen und Patienten mit und nach Herzstillstand notwendig ist, ist in Uganda bereits vorhanden bzw. etabliert. So gibt es derzeit beispielsweise nur eine Herzkatheter-Versorgungseinheit im ganzen Land. In vielen Gesprächen vor Ort mit Entscheidungsträgern konnten jedoch bereits erste Verbesserungen in Ausstattung und Ausrüstung angestoßen werden. Die relevanten Fachgesellschaften haben darüber hinaus eine Projektgruppe zur Gründung eines nationalen Rates für Wiederbelebung initiiert. Den größten Erfolg sieht Meyer jedoch darin, dass bei den Menschen vor Ort ein Feuer zum Thema Wiederbelebung entzündet werden konnte. Die Begeisterung bei Teilnehmern und Instruktoren ist riesengroß und macht Lust auf mehr.

\section{Unsere nächsten Kurstermine (Stand 31.03.2020)*}

GRC •

\section{Advanced Life Support Provider Kurs (ALS)}

Berlin

Aachen (leider bereits ausgebucht)

Lübeck

Aachen

Leipzig

Fürth

Mainz

Oldenburg

02.-03.05.2020

07.-08.05.2020

08. -10.05 .2020$

09. -10.05 .2020$

09.- 10.05 .2020

12.- 13.05 .2020

23. -24.05 .2020$

12.- 14.06 .2020

\section{ALS Refresher Kurse}

Landau

21.06 .2020

\section{Basic Life Support (BLS) und AED Provider Kurs}

Fürth

19.05.2020

\section{European Paediatric Advanced Life Support Provider Kurs}

(EPALS)

Ulm

22. -24.05 .2020$

Berlin

05.-07.06.2020

Generic Instructor Course (GIC)

Fürth

23. -24.05 .2020$

Newborn Life Support (NLS)

Stuttgart

13.05.2020

Fürth

26.05 .2020

Viersen

27.05.2020

${ }^{\star}$ Aufgrund der aktuellen Situation werden leider nicht alle Kurse stattfinden können. Bitte schauen Sie regelmäßig auf unsere Website und informieren Sie sich dort: https://www.grc-org.de/kurse/kurstermine. Bei allgemeinen Fragen zu den Kursen wenden Sie sich bitte gerne an sekretariat@grc-akademie.de.

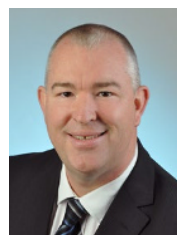

$\Delta$ Bernhard

Gliwitzky,

Geschäftsfüh-

rer der GRC

Kursakademie 